\title{
Impact of strong disorder on the static magnetic properties of the spin-chain compound $\mathrm{BaCu}_{2} \mathrm{SiGeO}_{7}$
}

\author{
T. Shiroka, ${ }^{1,2}$ F. Casola, ${ }^{1,2, *}$ W. Lorenz ${ }^{3}$ K. Prša, ${ }^{4}$ A. Zheludev, ${ }^{3}$ H.-R. Ott, ${ }^{1,2}$ and J. Mesot ${ }^{1,2}$ \\ ${ }^{1}$ Laboratorium für Festkörperphysik, ETH Hönggerberg, CH-8093 Zürich, Switzerland \\ ${ }^{2}$ Paul Scherrer Institut, CH-5232 Villigen PSI, Switzerland \\ ${ }^{3}$ Neutron Scattering and Magnetism, Laboratorium für Festkörperphysik, ETH Hönggerberg, CH-8093 Zürich, Switzerland \\ ${ }^{4}$ Institute of Condensed Matter Physics, EPFL, CH-1015 Lausanne, Switzerland
}

(Received 10 July 2013; published 26 August 2013)

\begin{abstract}
The disordered quasi-one-dimensional magnet $\mathrm{BaCu}_{2} \mathrm{SiGeO}_{7}$ is considered as one of the best physical realizations of the random Heisenberg chain model, which features an irregular distribution of the exchange parameters and whose ground state is predicted to be the scarcely investigated random-singlet state (RSS). Based on extensive ${ }^{29} \mathrm{Si} \mathrm{NMR}$ and magnetization studies of $\mathrm{BaCu}_{2} \mathrm{SiGeO}_{7}$, combined with numerical quantum Monte Carlo simulations, we obtain remarkable quantitative agreement with theoretical predictions of the random Heisenberg chain model and strong indications for the formation of a random-singlet state at low temperatures in this compound. As a local probe, NMR is a well-adapted technique for studying the magnetism of disordered systems. In this case, it also reveals an additional local transverse staggered field, which affects the low-temperature properties of the RSS. The proposed model Hamiltonian satisfactorily accounts for the temperature dependence of the NMR line shapes.
\end{abstract}

DOI: 10.1103/PhysRevB.88.054422

PACS number(s): 75.10.Pq, 76.60.-k, 75.10.Jm, 75.40.Cx

\section{INTRODUCTION}

Spin- $\frac{1}{2}$ Heisenberg chains adopt a nonmagnetic ground state, which qualitatively can be seen as a linear superposition of states representing all the possible ways of forming singlets in the system. ${ }^{1}$ With quantum fluctuations suppressing any long-range order, the translational symmetry is preserved. At large length scales (low energies), any amount of disorder in the exchange parameters is predicted to dominate over the quantum or thermal fluctuations ${ }^{2-4}$ and the resulting system is known as "random Heisenberg chain" (RHC). In practice, the disorder leads to an inhomogeneous ground state by associating to every random configuration of exchange paths a unique way of forming singlets, regardless of the distance between the involved spins and of their interactions. This new type of ground state, specific to random Heisenberg chains, is called random-singlet state (RSS). ${ }^{3}$

It is widely accepted ${ }^{4}$ that the interest in RHCs originally arose from a novel approach to deal with random exchanges in the isotropic spin- $\frac{1}{2}$ Heisenberg model, introduced in 1979 by Dasgupta and $\mathrm{Ma}^{2}$ Their new physical insight into the effects of disorder was later developed into a formal theory by Fisher $^{3}$ and, subsequently, applied to a large variety of problems involving magnets with quenched disorder. ${ }^{4-7}$ However, it is only the recent combined availability of materials representing physical realizations of disordered quasi-one-dimensional (quasi-1D) quantum magnets ${ }^{8-10}$ and of novel stochastic numerical methods ${ }^{11}$ that made possible the first quantitative studies concerning the impact of disorder on materials whose magnetic properties are well described by an antiferromagnetic Heisenberg-chain Hamiltonian [see Eq. (1)].

The aim of this work is to identify a model material featuring the properties of a RHC and, through experimental and numerical methods, to demonstrate that the chosen RHC Hamiltonian describes its physical behavior. Here, we present a set of field- and temperature-dependent magnetization and ${ }^{29} \mathrm{Si}$ nuclear magnetic resonance (NMR) data of the insulating material $\mathrm{BaCu}_{2} \mathrm{SiGeO}_{7} .{ }^{12}$ Its relevant structural unit is shown in Fig. 1 and its physical properties can be discussed in terms of the one-dimensional Hamiltonian

$$
\begin{aligned}
\mathscr{H}= & \sum_{i}\left[J_{x y}(i)\left(S_{i}^{x} S_{i+1}^{x}+S_{i}^{y} S_{i+1}^{y}\right)+J_{z}(i) S_{i}^{z} S_{i+1}^{z}\right] \\
& -g \mu_{\mathrm{B}} \sum_{i} H S_{i}^{z}+\mu_{\mathrm{B}} H_{\perp} \sum_{i}(-1)^{i} S_{i}^{x} .
\end{aligned}
$$

Here, $J_{x y}(i)=J_{z}(i)=J_{i}>0$ represent the random exchange couplings along the spin-chain sites $i, \mu_{\mathrm{B}}$ is the Bohr magneton, $H$ the uniform applied magnetic field, $H_{\perp}=c H$ a locally induced transverse staggered field, ${ }^{13}$ and the spin operators refer to a spin $S=\frac{1}{2}$.

In order to achieve a description of the large length scale/low-energy physics contained in Eq. (1) with $H=0$, Dasgupta and Ma developed a real-space renormalization procedure. They started from the local bonds rather than from the large spatial blocks, as is usually done for homogeneous systems. ${ }^{14}$ Starting from the distribution of unrenormalized exchange couplings $P\left(J_{i}, J\right)$, where $J$ is the energy cutoff, an effective low-energy theory is constructed by tracking, via flow equations, the behavior of $P\left(J_{i}, J\right)$ as the cutoff $J \sim k_{\mathrm{B}} T$ is progressively reduced. In the RSS theory, the flow of the distribution of unrenormalized bond energies $P\left(J_{i}\right)$ depends on the considered energy scale and temperature. ${ }^{2,3}$ As a result, the static magnetic properties are dominated by a temperature-dependent population of effectively paramagnetic moments $n(T)$. Their characteristic energy $(\omega)$ and temperature-dependent average spatial separation $\xi(T, \omega=$ $0) \sim n^{-1}(T)=\ln ^{2}\left(J_{0} / k_{\mathrm{B}} T\right)$ diverges as $T \rightarrow 0$, leading to a quasi-long-range ordered ground state. ${ }^{3}$ In the expression for $n^{-1}(T), J_{0}$ is the largest exchange coupling in the chain prior to the renormalization. ${ }^{15}$ Due to weak residual interchain interactions, a magnetic order at a Néel temperature $T_{\mathrm{N}}>0 \mathrm{~K}$ is established also in the random Heisenberg chains, albeit with a lower ordering temperature in comparison with the 


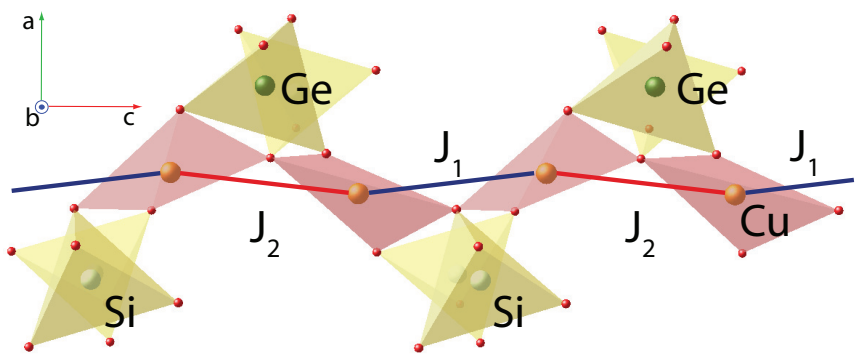

FIG. 1. (Color online) Sketch of the relevant structural unit in $\mathrm{BaCu}_{2} \mathrm{SiGeO}_{7}$ highlighting the different $J_{1}$ and $J_{2}$ exchange couplings. Spin chains run along the crystallographic $c$ axis.

disorder-free case. ${ }^{16,17}$ In this work, we focus our attention on the local and bulk static magnetic properties of $\mathrm{BaCu}_{2} \mathrm{SiGeO}_{7}$ for $T>T_{\mathrm{N}}$ and $H \neq 0$.

\section{II. $\mathrm{BaCu}_{2} \mathrm{SiGeO}_{7}$ : SUMMARY OF PREVIOUS RESEARCH}

The experimental work on $\mathrm{BaCu}_{2} \mathrm{SiGeO}_{7}$ began with studies on tunable superexchange interactions in spin-chain systems by Yamada et al. ${ }^{12}$ Across the series from $\mathrm{BaCu}_{2} \mathrm{Si}_{2} \mathrm{O}_{7}$ to $\mathrm{BaCu}_{2} \mathrm{Ge}_{2} \mathrm{O}_{7}$, the $\mathrm{BaCu}_{2}\left(\mathrm{Si}_{1-x} \mathrm{Ge}_{x}\right)_{2} \mathrm{O}_{7}$ compounds $(0<$ $x<1)$ crystallize with a Pnma space group. For $x=0.5$, corresponding to maximum disorder in the exchange couplings, the values of the lattice parameters are $a=6.917$ (7) $\AA, b=$ 13.28 $\AA$, and $c=6.944$ (7) $\AA,{ }^{18}$ in-between the parameters of the two end members of the series. The spin chains run along the crystallographic $c$ axis, as shown in Fig. 1.

Early magnetization measurements of polycrystalline samples revealed the often-observed broad maximum of $\chi(T)$, also known as the Bonner-Fisher peak. ${ }^{19}$ This maximum shifts linearly with $x$ towards higher temperatures as the Ge concentration is enhanced. From this feature it was initially deduced that the high-temperature properties of $\mathrm{BaCu}_{2} \mathrm{SiGeO}_{7}$ effectively reflect those of a standard spin- $\frac{1}{2}$ Heisenberg chain with $J_{\text {eff }}=\left(J_{\mathrm{Si}}+J_{\mathrm{Ge}}\right) / 2 \approx 37 \mathrm{meV}$, where $J_{\mathrm{Si}}=24.1 \mathrm{meV}$ and $J_{\mathrm{Ge}}=50 \mathrm{meV}$ represent the intrachain exchange interactions for no and for complete Ge substitution, respectively. The enhancement of the AFM exchange, resulting from a higher Ge content, was explained in terms of a change of the $\mathrm{Cu}-\mathrm{O}-\mathrm{Cu}$ bonding angle, from $\phi=124^{\circ}$ in $\mathrm{BaCu}_{2} \mathrm{Si}_{2} \mathrm{O}_{7}$ to $135^{\circ}$ in the Ge parent compound. ${ }^{20}$

Earlier bulk measurements already revealed two distinct features: $:^{12}$ (i) a low-temperature divergence of the spin susceptibility in $\mathrm{BaCu}_{2} \mathrm{SiGeO}_{7}$, interpreted as a simple Curie-type behavior due to uncompensated magnetic moments, ${ }^{21}$ and (ii) a low-temperature magnetic order, expected even in the case of random Heisenberg chains (RHCs), ${ }^{17}$ with an onset at $T_{\mathrm{N}}=0.7 \mathrm{~K}$ in $\mathrm{BaCu}_{2} \mathrm{SiGeO}_{7} .{ }^{18}$

In zero magnetic field, $\mathrm{BaCu}_{2} \mathrm{Si}_{2} \mathrm{O}_{7}$ is known to order antiferromagnetically at $T_{\mathrm{N}}=9.2 \mathrm{~K} .{ }^{22}$ The more than tenfold reduction of $T_{\mathrm{N}}$ in $\mathrm{BaCu}_{2} \mathrm{SiGeO}_{7}$ may be related to the fact that the introduction of Ge modifies not only the intrachain, but also the interchain coupling which, along the $a$ axis, changes from ferromagnetic (FM) to antiferromagnetic (AFM) type. ${ }^{12}$ The latter implies that for $\mathrm{BaCu}_{2} \mathrm{SiGeO}_{7}$ the interchain coupling alternates in sign and, therefore, it averages out to a mean-field value. $^{23}$
The interest in $\mathrm{BaCu}_{2} \mathrm{SiGeO}_{7}$ grew significantly as soon as results of magnetic-susceptibility measurements on single crystals appeared in the literature. ${ }^{21}$ They suggested a logarithmic dependence of the low-temperature spin susceptibility, ${ }^{21}$ consistent with predictions of the RHC model. ${ }^{2}$ Subsequent inelastic neutron-scattering (INS) studies focused on the measurement of the energy dependence of the correlation length $\xi(T=0, \omega)$, which at zero temperature is inversely proportional to the free-spin concentration $\xi(\hbar \omega) \sim n^{-1}(\hbar \omega)=$ $\ln ^{2}\left(J_{0} / \hbar \omega\right)$, with $\hbar \omega$ the energy of the probing neutrons. In INS, $\xi$ is extracted from the inverse width of the energy-integrated scattering intensity $S(\mathbf{q})$ which, in case of a disordered phase, is typically of Lorentzian shape. ${ }^{24}$ Following some earlier misinterpretation of the data due to problems with background subtraction, ${ }^{21,25}$ the INS results were found to be quantitatively consistent with a Luttinger-liquid (LL) behavior reflecting a disorder-free spin chain with a single effective coupling $J_{\text {eff }} \approx 37 \mathrm{meV}^{18}$ It was concluded that the expected RHCrelated physics must manifest itself at energies much lower than those accessible by neutron experiments. In a previous NMR study, ${ }^{15}$ we already pointed out how the spin dynamics of $\mathrm{BaCu}_{2} \mathrm{SiGeO}_{7}$, probed at the characteristic Larmor energy $\hbar \omega_{\mathrm{L}} \sim 1 \mu \mathrm{eV}$ (with $\omega_{\mathrm{L}} / 2 \pi$ the NMR resonance frequency), turns out to be notably different with respect to that of the regular Heisenberg chain $\mathrm{BaCu}_{2} \mathrm{Si}_{2} \mathrm{O}_{7}$. On the other hand, considering existing data on $\mathrm{BaCu}_{2} \mathrm{Si}_{2} \mathrm{O}_{7}$ and $\mathrm{BaCu}_{2} \mathrm{Ge}_{2} \mathrm{O}_{7}$, a low-temperature divergence of the magnetic susceptibility also in $\mathrm{BaCu}_{2} \mathrm{SiGeO}_{7}$ is not really surprising. ${ }^{13}$ In addition, since INS failed to detect any sort of disorder-induced spin dynamics in this compound, the existence of a random-singlet state (RSS, the ground state of the isotropic RHC Hamiltonian) was considered questionable. In this work, we show how the effect of disorder on the static magnetic properties can be quantitatively modeled and compared with experiments, thereby supporting the RHC scenario.

\section{MAGNETIC SUSCEPTIBILITY OF $\mathrm{BaCu}_{2} \mathrm{SiGeO}_{7}$}

\section{A. Experimental results}

As a first step, we extended the measurements of the magnetic susceptibility $\chi(T, H)$ to wider temperature and field ranges than those covered in previous studies. Single crystals of $\mathrm{BaCu}_{2} \mathrm{SiGeO}_{7}$ were grown by the floating-zone technique. One of them was cut and the smaller $6.5-\mathrm{mg}$ part was used in magnetometry measurements; the remaining 20-mg piece was used for the NMR investigations.

The magnetization of the chosen sample was measured by applying a magnetic field $\mathbf{H} \| b$. Magnetic susceptibility data $\chi=M / H$ are displayed as bold solid lines in Fig. 2. For $\mu_{0} H=0.1 \mathrm{~T}$, and at temperatures $T \geqslant 1.8 \mathrm{~K}$, the measurements were performed using a standard dc SQUID magnetometer, whereas to cover temperatures down to $0.5 \mathrm{~K}$ we employed a SQUID device with a ${ }^{3} \mathrm{He}$ insert. For all the other fields and for temperatures $T \geqslant 1.8 \mathrm{~K}$, we used a vibrating-sample magnetometer (VSM). Since in the latter case data are collected "on the fly," i.e., while the temperature is being swept at $0.25 \mathrm{~K} / \mathrm{min}$, this causes a small (known) temperature difference between sample and thermometer position. In addition, a certain dispersion in the magnetization 


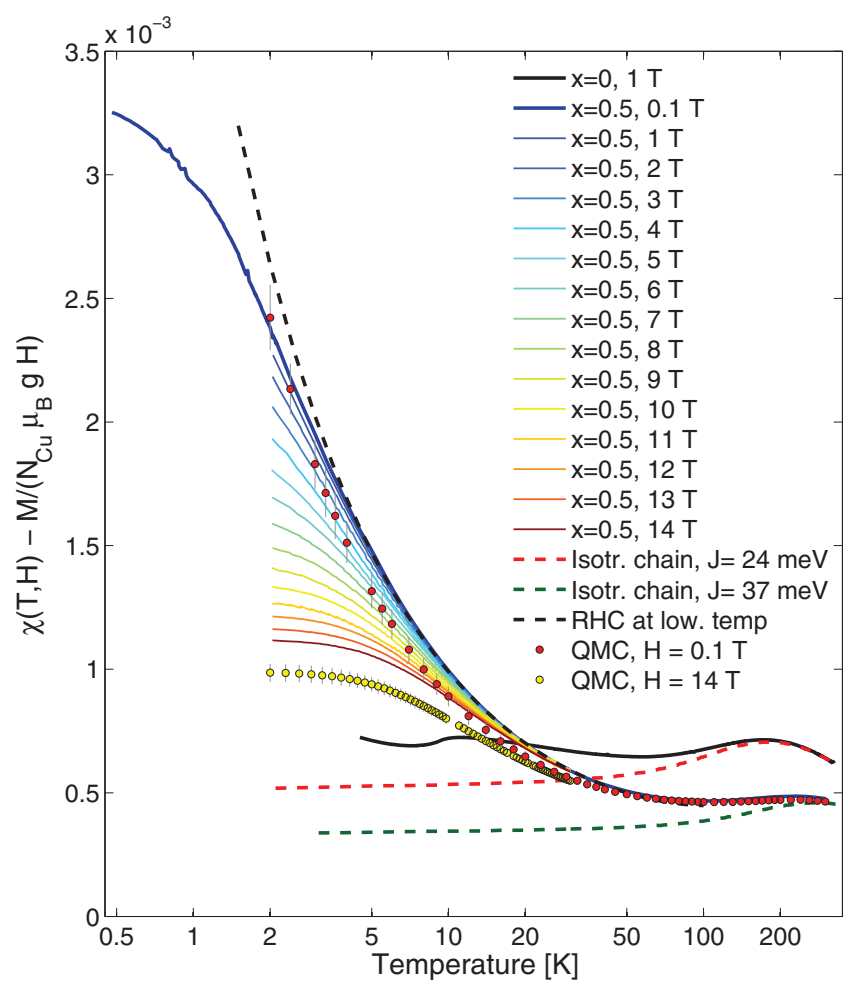

FIG. 2. (Color online) Field and temperature dependencies of the magnetic susceptibility $\chi(T, H)$ per $\mathrm{Cu}$ ion in $\mathrm{BaCu}_{2} \mathrm{SiGeO}_{7}$ (and in $\mathrm{BaCu}_{2} \mathrm{Si}_{2} \mathrm{O}_{7}$, at $1 \mathrm{~T}$ ) with $\mathbf{H} \| b$ in normalized units, assuming $g=2$ (Ref. 13). The fit of $\chi(T, 0.1 \mathrm{~T})$ with a RHC model was adopted from Ref. 3, while the $\chi(T)$ curves for chains with a single isotropic exchange value were calculated as in Ref. 26. QMC simulations of the susceptibility $\chi(T, H)$ at $\mu_{0} H=0.1$ and $14 \mathrm{~T}$ for a simple $J_{1}-J_{2}$ RHC model is also shown (see text for details).

values was reduced by applying a moving-average filter with a $200-\mathrm{mK}$ width to the raw experimental data. Magnetic hysteresis, defined as the difference between the zero-fieldcooled and field-cooled magnetization data, was observed for $T<800 \mathrm{mK}$. The hysteretic behavior is probably related to ferromagnetic domains with a rather small net magnetic moment present in the ordered low-temperature phase. Since only the zero-field-cooled scans are relevant for our discussion, the field-cooled data are not shown.

\section{B. Data analysis}

As shown in Fig. 2, for temperatures above $30 \mathrm{~K}$ the $\chi(T, H)$ curves are field independent and, for $T>200 \mathrm{~K}$, they approach the theoretical prediction for an isotropic Heisenberg chain $^{26}$ with an exchange parameter $J_{\text {eff }}=\left(J_{\mathrm{Si}}+J_{\mathrm{Ge}}\right) / 2 \approx$ $37 \mathrm{meV}$ (see the green dashed line in Fig. 2). For comparison, the red dashed line indicates the calculated $\chi(T)$ for an isotropic chain with $J_{\mathrm{Si}} \approx 24 \mathrm{meV}$, corresponding to the $x=0$ case.

We first discuss two major differences between the magnetic susceptibilities of $\mathrm{BaCu}_{2} \mathrm{Si}_{2} \mathrm{O}_{7} \quad(x=0)$ and $\mathrm{BaCu}_{2} \mathrm{SiGeO}_{7}(x=0.5)$ : (i) Our previous successful attempt to interpret the low-temperature increase/divergence of $\chi(T)$ in $\mathrm{BaCu}_{2} \mathrm{Si}_{2} \mathrm{O}_{7}$ and $\mathrm{BaCu}_{2} \mathrm{Ge}_{2} \mathrm{O}_{7}$ as simply being caused by a local transverse staggered field [LTSF (Ref. 13), see
Sec. IV B] is inadequate for treating the more complex case of $\mathrm{BaCu}_{2} \mathrm{SiGeO}_{7}$. First of all, the magnetic susceptibility $\chi(T<10 \mathrm{~K}, H)$ is strongly reduced even by moderate fields. As a result, the magnetization deviates from the linear response with respect to $H$ (see Fig. 7 and Ref. 26), expected either for an isotropic chain in the LL regime, or for the sine-Gordon (SG) model in the high-temperature limit, the latter describing the physics when an LTSF is present. ${ }^{13}$

The SG model, even at intermediate temperatures, is inadequate to describe the physics of $\mathrm{BaCu}_{2} \mathrm{SiGeO}_{7}$. Indeed, in this case $\chi(T)$ is known to exhibit a symmetric peak centered at a temperature $T_{m}$, roughly corresponding to half of the field-induced gap $\Delta_{\mathrm{SG}}{ }^{27}$ To exemplify this fact, we consider the Hamiltonian in Eq. (1) with $J_{i}=J$ and $H \neq 0$. In the reduced units $h_{i, \perp}^{*}=\mu_{\mathrm{B}} H_{i, \perp} / J_{\text {eff }}$, with $H_{i, \perp}=(-1)^{i} c H$, this model features a gap $\Delta_{\mathrm{SG}}$ in the spin-excitation spectrum which, for $h_{\perp}^{*} \ll 1$, scales as ${ }^{28}$

$$
\frac{\Delta_{\mathrm{SG}}}{J}=1.78 \times\left(h_{\perp}^{*}\right)^{\frac{2}{3}}\left(-\ln h_{\perp}^{*}\right)^{\frac{1}{6}} .
$$

Equation (2) implies that for $c=0.092$ [the value estimated for $\mathrm{BaCu}_{2} \mathrm{Si}_{2} \mathrm{O}_{7}$ (Ref. 13) if $\mathbf{H} \| b$ ], the LTSF yields $\Delta_{\mathrm{SG}} \simeq$ $1.42 \mathrm{meV}$ at $H=14 \mathrm{~T}$, corresponding to $T_{m} \simeq 8.2 \mathrm{~K}$. On the other hand, as can be seen in Fig. 2, the susceptibility recorded at $14 \mathrm{~T}$ tends to saturate at low temperatures, rather than display a peak at $T_{m}$.

(ii) The inadequacy of the SG model in interpreting the $\chi(T, H)$ data of $\mathrm{BaCu}_{2} \mathrm{SiGeO}_{7}$ is particularly evident from the $(T, H)$ dependence of $T_{i}$, the inflection point which marks the start of saturation [corresponding to the maximum of $|\partial M / \partial T|$ in a fixed field $H$ (see Fig. 3)]. To overcome noise problems in the calculation of $|\partial M / \partial T|$ from raw magnetization data, the experimental points were linearly interpolated using a $200-\mathrm{mK}$ binning and the resulting line slope was taken as the derivative with respect to temperature.

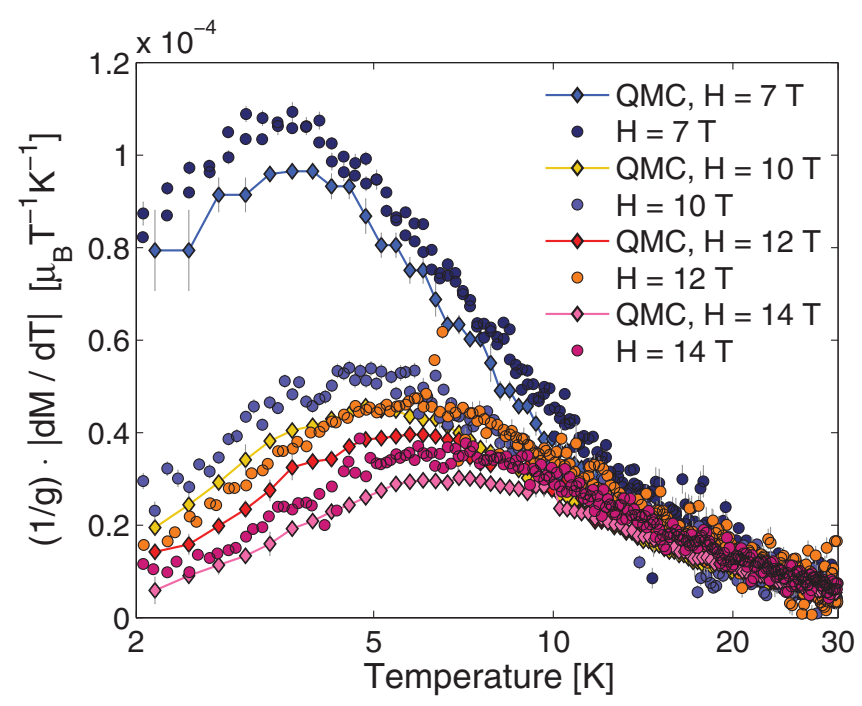

FIG. 3. (Color online) First derivative of the sample magnetization vs temperature, $|\partial M(T, H) / \partial T|$, in $\mathrm{BaCu}_{2} \mathrm{SiGeO}_{7}$ for $H \| b$. The graphic compares several cross sections of the color map shown in Fig. 4. The assigned errors reflect the line-slope uncertainty for fits within the binning range (see text for details). The shown QMC simulation results were obtained without using any free parameters. 

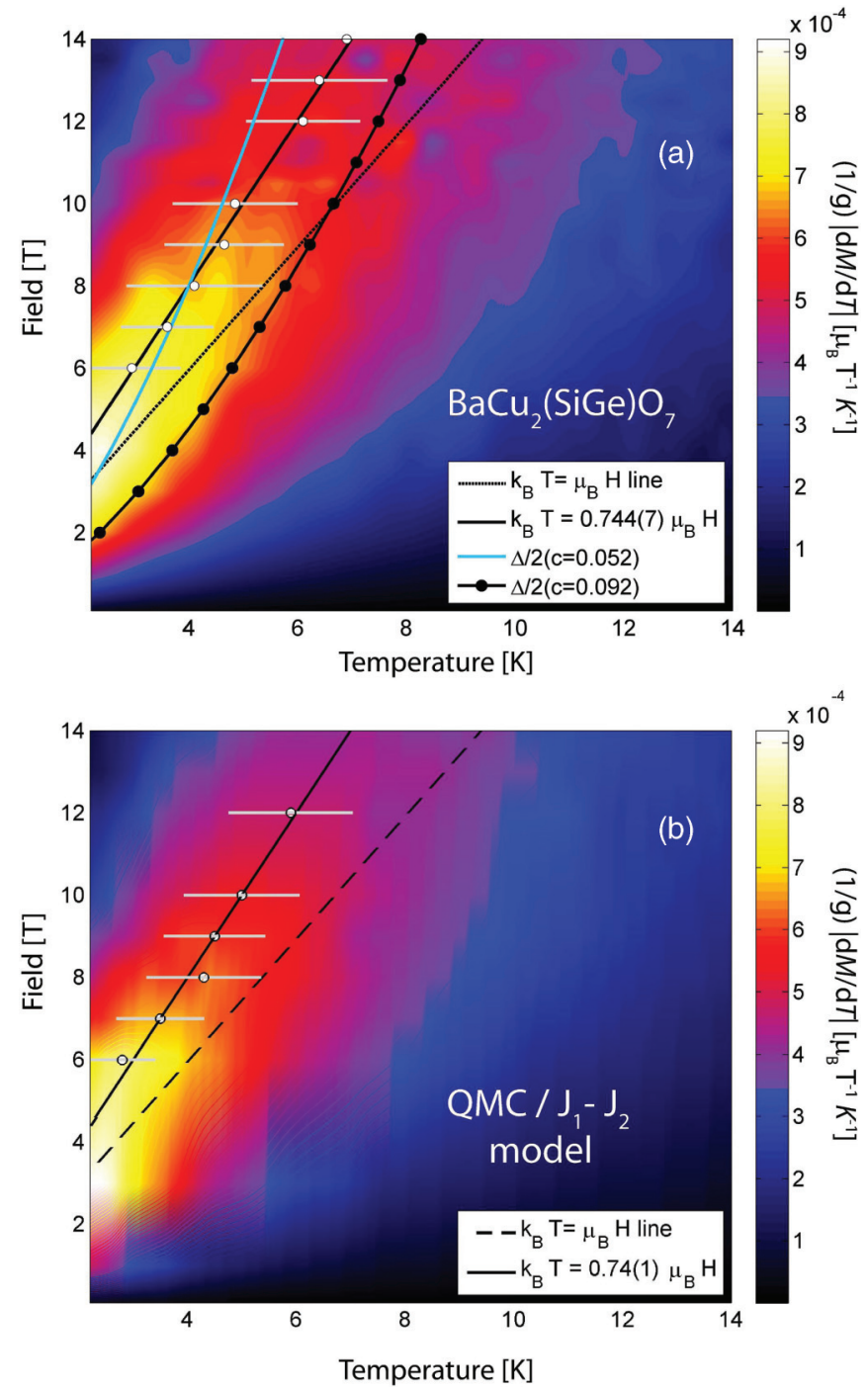

FIG. 4. (Color online) (a) First derivative of the sample magnetization vs temperature, $|\partial M(T, H) / \partial T|$, in $\mathrm{BaCu}_{2} \mathrm{SiGeO}_{7}$ for $H \| b$ (data scaled using $g=2$ ) (Ref. 13). The open circles represent crossover temperatures. For details on data treatment and for a description of the plotted lines, see text. (b) QMC simulation of the $J_{1}-J_{2}$ RHC model for a system of 6000 spins, using 20 realizations of disorder for each $(T, H)$ point. The color map was generated using the same data treatment as in panel (a).

The results of this procedure are shown as colored circles in Fig. 3 for four selected fields. A $(T, H)$ color map of the $|\partial M / \partial T|$ values is shown in Fig. 4(a). Here, the inflection points (assigned with an error corresponding to the peak width at $95 \%$ of the peak height in Fig. 3) are shown as open circles. The resulting $T_{i}(H)$ values follow a linear field dependence, such that $k_{\mathrm{B}} T_{i}=0.744(7) \mu_{\mathrm{B}} H$. Aside from requiring a nonuniversal dimensionless prefactor, ${ }^{29}$ the field dependence of $T_{i}$ does not follow the $H^{2 / 3}$ power law that the small $h_{i, \perp}^{*}$ value would imply for $\Delta(H) / 2 .{ }^{27}$ The calculated dependencies of $\Delta / 2$ for $H_{\perp}=(-1)^{i} c H$ with $c=0.092$ or 0.052 are both also shown in Fig. 4(a). The first $c$ value represents the LTSF proportionality constant in $\mathrm{BaCu}_{2} \mathrm{Si}_{2} \mathrm{O}_{7} \cdot{ }^{13}$ Because of the similar local environments, it seems reasonable to use the same value also for $\mathrm{BaCu}_{2} \mathrm{SiGeO}_{7}$. The reason for choosing $c=0.052$ instead will be clarified in Sec. IV.

In view of these two inadequacies, we modified our previous approach to the experimental data analysis ${ }^{13}$ and instead chose to compare the experimental results with the predictions of the RHC model Hamiltonian in Eq. (1). ${ }^{3}$ A first comparison is carried out by fixing $c=0$ and by assuming the $J_{i}$ values to be uniformly distributed between the two limits $J_{\mathrm{Si}}$ and $J_{\mathrm{Ge}}$.

In case of nonzero temperatures and magnetic fields, the renormalization flow of the decimation procedure is interrupted either at the thermal energy $k_{\mathrm{B}} T$, or at the magnetic energy $g \mu_{\mathrm{B}}\langle S\rangle H .{ }^{29}$ In the first case, the resulting susceptibility is $^{3}$

$$
\chi(T)_{\mathrm{RHC}} \sim T^{-1} \ln ^{-2}\left(J_{0} / k_{\mathrm{B}} T\right) .
$$

In the second case, the system is driven away from the zerofield fixed point into a regime with field-aligned undecimated spins, a saturated magnetization, and zero entropy. ${ }^{29}$ In spin$\frac{1}{2}$ AFM RHCs, the decimation procedure involving the total set of spins leaves the renormalized magnetization magnitude at $\langle S\rangle=\frac{1}{2}$, unlike, for example, in a RHC with FM-AFM coupling. ${ }^{29}$ For this reason, the onset of saturation of the lowtemperature magnetization occurs at $k_{\mathrm{B}} T_{i} \approx \mu_{\mathrm{B}} H \cdot{ }^{29}$ A fit to the susceptibility data at the smallest applied field, using the theoretical random-singlet expression for $\chi(T)_{\mathrm{RHC}}$, is shown in Fig. 2 as a black dashed line. A rather good match is found above $4 \mathrm{~K}$ with a cutoff $J_{0} \simeq 66.3 \pm 0.7 \mathrm{meV}$.

To achieve a more quantitative comparison, we carried out a series of quantum Monte Carlo (QMC) simulations based on Eq. (1), where $J_{i}$ couplings alternate randomly from $J_{1}=J_{\mathrm{Si}}=24.1 \mathrm{meV}$ to $J_{2}=J_{\mathrm{Ge}}=50 \mathrm{meV}$ (we call this a $J_{1}-J_{2}$ model), mimicking the situation expected for $\mathrm{BaCu}_{2} \mathrm{SiGeO}_{7}$. We chose 6000 spin sites with randomly distributed but equally probable $J_{1}$ and $J_{2}$ couplings. The simulations for $\chi(T, H)$ were averaged over at least 25 random realizations of disorder, as obtained from a directed-loop algorithm ${ }^{30}$ within the ALPS 2.0 package. ${ }^{11}$ Technical details related to the QMC simulations are discussed in the Appendix. Disorder in the exchange couplings was imposed by assuming an equal number ( $L / 2$, with $L$ as the chain length) of $J_{1}$ and $J_{2}$ values, randomly permuted to construct the RHC chain that was to be simulated.

Selected results of these simulations are shown as red and yellow circles in Fig. 2, as interconnected diamonds in Fig. 3, and as a color map in Fig. 4(b). In view of the simplicity of the model, the qualitative and quantitative agreement of our parameter-free QMC simulations with the data is remarkable. Incidentally, the best fit of the RS prediction to the low- $T$ QMC results in an applied field $\mu_{0} H=0.1 \mathrm{~T}$ requires $J_{0} \approx J_{2}$. This corresponds to the largest energy scale in the system before the decimation starts, thus reinforcing the pertinence of the theory used to capture the magnetic properties of the system. As may be seen in Figs. 3 and 4(b), by applying the moving-average procedure described above also to the QMC results, numerical calculations predict the same free-spin-like linear field dependence for $T_{i}$ as observed experimentally. This is an important result because the existence of paramagnetic entities at $T=10 \mathrm{~K}$ in a strongly correlated system, with a characteristic energy scale 
corresponding to a temperature exceeding $200 \mathrm{~K}$, is highly counterintuitive.

\section{NUCLEAR MAGNETIC RESONANCE IN A RANDOM HEISENBERG CHAIN}

Although the measurements of $\chi(T, H)$ and the comparison with QMC calculations already provide a strong indication for the formation of a $\mathrm{RS}$ phase in $\mathrm{BaCu}_{2} \mathrm{SiGeO}_{7}$ at low temperatures, it is tempting to directly verify the presence of a random-singlet state on a local scale. With decreasing temperature, an increasing number of spins form pairs by adopting a nonmagnetic singlet ground state. The divergent low-temperature tail of $\chi(T, H)$ is due to the residual unpaired spins. The tendency to form a random-singlet ground state should be directly manifest in NMR data, reflecting a growing number of ${ }^{29} \mathrm{Si}$ sites which experience a zero net transferred local field.

\section{A. Experimental results}

Height-normalized ${ }^{29} \mathrm{Si}$ NMR lines, as recorded in a 7-T external field applied along the crystallographic $b$ axis, are plotted in Fig. 5. The spectra were obtained by superposing

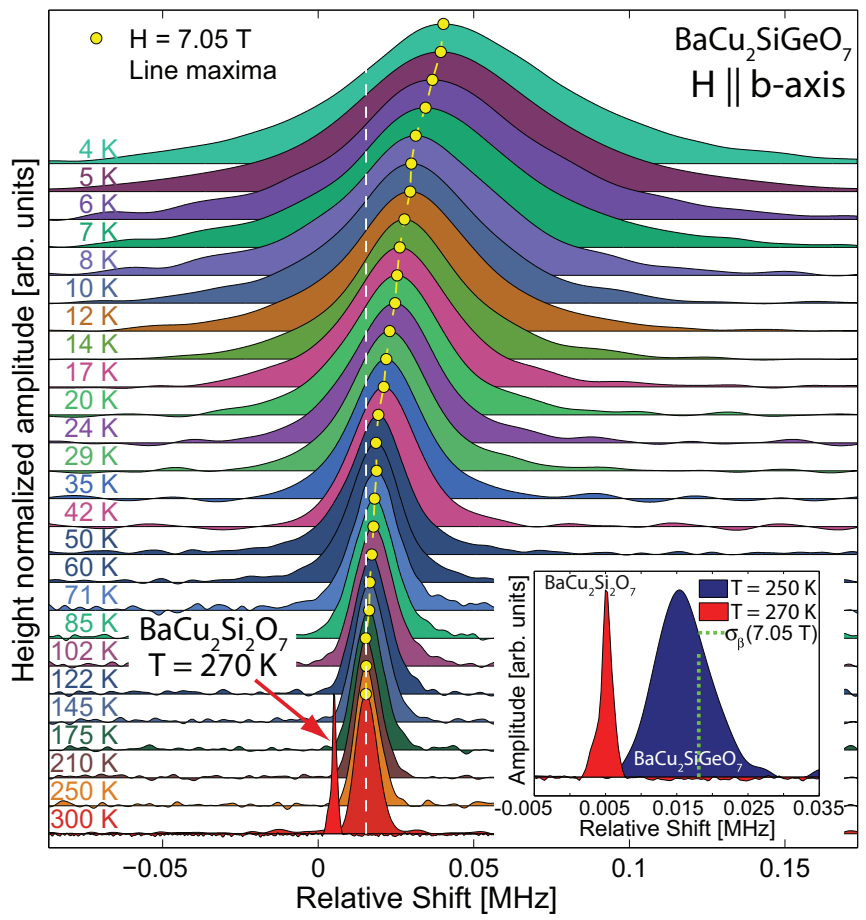

FIG. 5. (Color online) Temperature dependence of the ${ }^{29} \mathrm{Si}$ NMR lines of the disordered Heisenberg chain compound $\mathrm{BaCu}_{2} \mathrm{SiGeO}_{7}$ in a magnetic field of $7.05 \mathrm{~T}$ applied along the $b$ axis. The zero frequency marks the resonance of ${ }^{29} \mathrm{Si}$ nuclear spins in a standard reference sample such as $\mathrm{Si}\left(\mathrm{CH}_{3}\right)_{4}$ (Ref. 32). The white dashed line marks the position of the resonance at room temperature, while the yellow points represent the position of the maxima, displaying a shift towards higher frequencies at low temperatures. The inset compares the cases of maximum and no disorder at high temperature. The green dashed line marks the orbital shift as obtained from Ref. 13 (see text for details). several acquisitions in the frequency-sweep mode, as described in Ref. 31.

The effects of disorder are evident already by comparing the high-temperature line shapes of $\mathrm{BaCu}_{2} \mathrm{SiGeO}_{7}$ and $\mathrm{BaCu}_{2} \mathrm{Si}_{2} \mathrm{O}_{7}$ (see inset of Fig. 5). We note that the ${ }^{29} \mathrm{Si}$ resonance of $\mathrm{BaCu}_{2} \mathrm{SiGeO}_{7}$, apart from being broader than that of its pristine counterpart, is also shifted to higher frequencies. We recall that NMR data of $\mathrm{BaCu}_{2} \mathrm{Si}_{2} \mathrm{O}_{7}$ (taken at $\mu_{0} H=7 \mathrm{~T}$, with $\mathbf{H} \| b$ ) exhibit a positive orbital shift of $\sigma_{b} \simeq 0.018 \mathrm{MHz}$, with a negative hyperfine coupling to the longitudinal magnetization. ${ }^{13}$ As shown in Fig. 2, the hightemperature magnetization of $\mathrm{BaCu}_{2} \mathrm{SiGeO}_{7}$ is equivalent to that of an isotropic chain with $J=37 \mathrm{meV}$ and is distinctly smaller than that of $\mathrm{BaCu}_{2} \mathrm{Si}_{2} \mathrm{O}_{7}$, well modeled by choosing $J=24.1 \mathrm{meV}$. Consequently, the negative hyperfine shift in case of a sample with disorder is smaller and, hence, the resonance is located closer to $\sigma_{b}$ (see inset of Fig. 5). This result is ultimately an independent confirmation of the validity of the model which allowed us to extract the parameter $\sigma_{b}$ in Ref. 13. We emphasize that, due to a random variation of the hyperfine interactions, a broadening of the resonance in the $x=0.5$ case may well be of structural origin, rather than due to magnetic disorder. Realistically, both structural and magnetic disorder have to be taken into account and one of the aims of the analysis outlined below is to disentangle them. Another striking feature of the NMR signals is the temperature-dependent shift of the line maxima. In Fig. 5, this shift is evidenced by comparing the sequence of yellow points, marking the maxima, with the vertical dashed line, representing the peak position of the $300-\mathrm{K}$ line. It indicates a growing nonzero uniform magnetization as the temperature is lowered, in obvious contradiction to the RS-phase hypothesis, where the formation of singlets would imply the absence of a local magnetization. As expected, unlike what is observed in $\mathrm{BaCu}_{2} \mathrm{Si}_{2} \mathrm{O}_{7},{ }^{13,22}$ the NMR data show no indications of a phase transition around $T=10 \mathrm{~K}$ for the disordered compound.

\section{B. Data analysis}

In order to interpret the recorded NMR line shapes and positions, we compare the experimental data with the results of QMC simulations of the $J_{1}-J_{2}$ model, addressed in Sec. III. In Fig. 6(a), we show the temperature-dependent, height-normalized histograms, which reflect the occurrences of longitudinal local magnetization values $S_{i}^{z}$ in $10^{-3} \mu_{\mathrm{B}}$ intervals for $\mu_{0} H=7 \mathrm{~T}$ ( $z$ is the spin quantization axis along which the external field was applied). The histograms were obtained by using a single configuration of disorder in a system of $L=6000$ spins. We note that at $T=300 \mathrm{~K}$ the $S_{i}^{z}$ values are distributed around a nonzero, albeit small, uniform magnetization. In this regime, the details of disorder are not essential for describing the physics. At low temperatures, however, a broadened magnetization peak appears, centered at zero local moment, as expected in the case of a continuous formation of singlets. At the same time, we observe weak but extremely broad tails [see top histogram in Fig. 6(a)], indicating the presence of incompletely compensated spins, which are progressively polarized as the temperature is reduced.

As shown in Fig. 6(b), the local-moment distribution obtained from these simulations (which do not include a LTSF) 

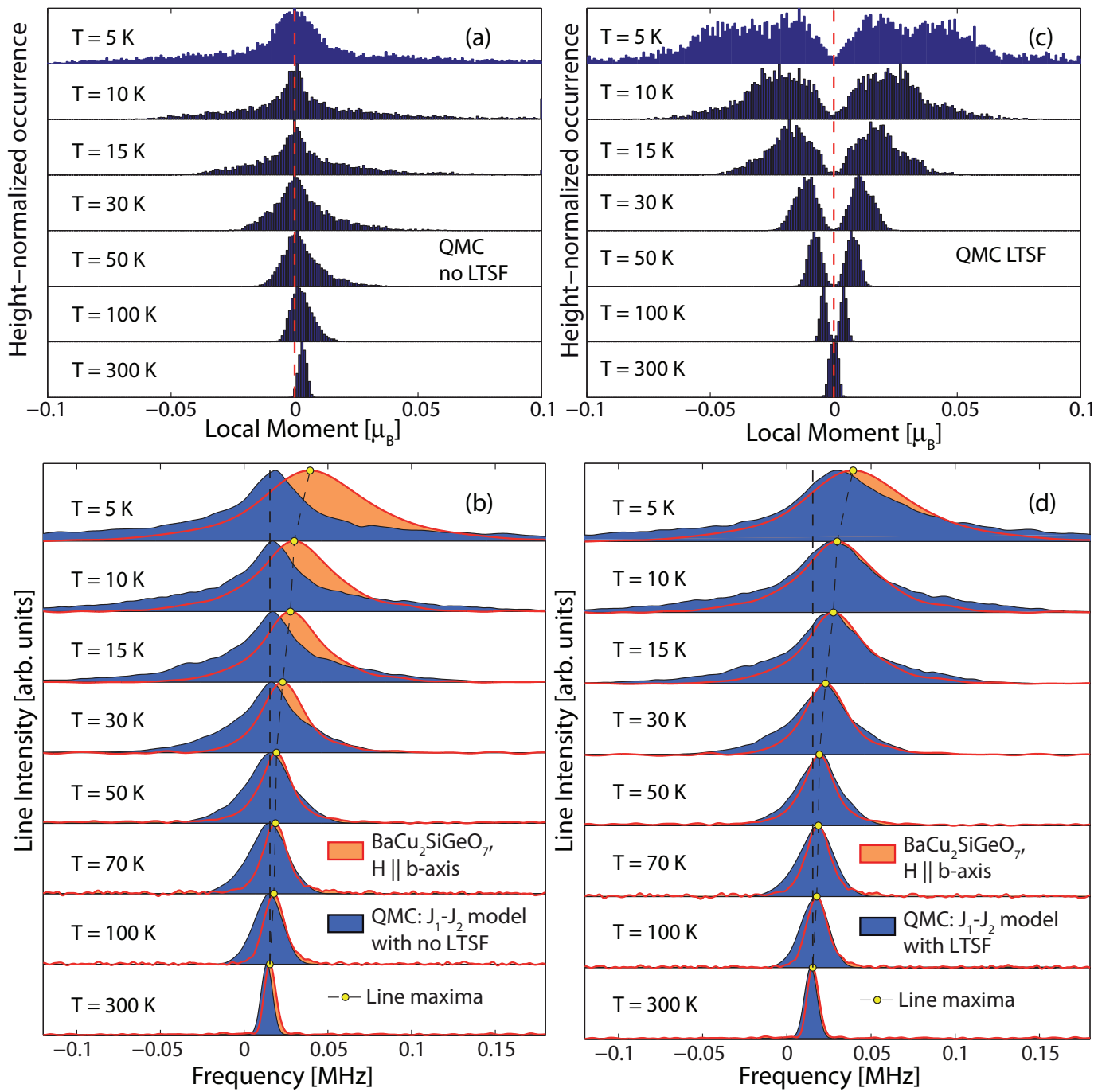

FIG. 6. (Color online) (a) QMC simulation of the local magnetization of a $J_{1}-J_{2}$ model of a spin chain with 6000 sites placed in an external field of $7 \mathrm{~T}$. The number of occurrences of the magnitude of a certain local moment is plotted, with each occurrence calculated in equidistant intervals of $10^{-3} \mu_{\mathrm{B}}$ and normalized to the most frequent one. The red dashed line marks the zero. (b) Simulated NMR line shapes (blue) using the results obtained from (a) and compared with the experimental data (orange). The black dashed line at fixed frequency marks the position of the peak of the $\mathrm{BaCu}_{2} \mathrm{SiGeO}_{7}$ line at $300 \mathrm{~K}$. No LTSF is present in this case. (c) Same as in (a), but with the uniform field replaced by a staggered field $H_{i, \perp}=(-1)^{i} c H$, with $c=0.092$ and $\mu_{0} H=7 \mathrm{~T}$. (d) Same as in (b), but including a LTSF in the model (see text for details).

does not match the experimental NMR data. The formation of random singlets implies that at a significant number of sites, the moments of the $\mathrm{Cu}$ atoms are quenched at low temperatures, corresponding to a zero shift of the simulated NMR line, in disagreement with observations. It turns out that it is essential to consider the combined effect of random exchange interactions and a LTSF, namely, to set $c \neq 0$ in the Hamiltonian (1). To include a LTSF, we use the same approximation made in the case of no disorder, ${ }^{13,27}$ i.e., we assume the total local magnetization $\mathbf{m}_{i}$ to be the sum of a nonuniform longitudinal and a transverse contribution, in the form

$$
\mathbf{m}_{i}=\left\langle\mathbf{S}_{i}^{\|}\right\rangle+\left\langle\mathbf{S}_{i}^{\perp}\right\rangle,
$$

where for the magnitude of the local transverse magnetization we write $\left\langle S_{i}^{\perp}\right\rangle\left(H, H_{i, \perp}\right) \approx\left\langle S_{i}^{\perp}\right\rangle\left(0, H_{i, \perp}\right)$, and analogously for the longitudinal component, with $H_{i, \perp}=c_{i} H \cdot{ }^{13}$ Because of this particular approximation, two QMC simulations, one for $S_{i}^{\perp}$ and another for $S_{i}^{\|}$, can be carried out separately on a diagonal basis. With $H \neq 0$, an LTSF acts on paired spins by mixing the nonmagnetic singlet states $|s\rangle$ with the magnetic triplet states $\left|t_{+1}\right\rangle=\left|\uparrow_{1} \uparrow_{2}\right\rangle$ and $\left|t_{-1}\right\rangle=\left|\downarrow_{1} \downarrow_{2}\right\rangle$, thereby inducing a nonzero magnetization also in the ground state of the spin pair. ${ }^{33}$ If both the uniform field and the LTSF are considered, in a first-order perturbation approximation, the new ground state can be written as ${ }^{33}$

$$
\begin{aligned}
& \left|s^{\prime}\right\rangle \approx|s\rangle+\alpha_{+}\left|t_{+1}\right\rangle-\alpha_{-}\left|t_{-1}\right\rangle, \quad \text { where } \\
& \alpha_{ \pm} \propto \frac{c H \mu_{\mathrm{B}}}{J \mp g \mu_{\mathrm{B}} H} .
\end{aligned}
$$

The uniform field appearing in the denominator can be neglected if $J \gg g \mu_{\mathrm{B}} H$, which is the case for the strongest coupled singlets in the RS phase. Therefore, at temperatures 
$k_{\mathrm{B}} T \approx \min (J) \gg g \mu_{\mathrm{B}} H$ the longitudinal local magnetization varies linearly with the applied field. Here, $\min (J)$ refers to the minimum exchange coupling value of the already formed singlets.

The distribution of local-magnetization values in a $J_{1}-J_{2}$ RHC model which includes a LTSF is shown in Fig. 6(c). Clearly, the presence of a LTSF substantially modifies the magnetization profiles shown in Fig. 6(a). By assuming that the local symmetry of the oxygen atoms surrounding a $\mathrm{Cu}^{2+}$ ion is largely unaffected by the Si-to-Ge substitution, the same value of $c=0.092$ obtained for $\mathrm{BaCu}_{2} \mathrm{Si}_{2} \mathrm{O}_{7}$ ought to be valid also in our case. As seen in Fig. 6(c), at $T=300 \mathrm{~K}$ and $\mu_{0} H=7 \mathrm{~T}$, the chosen LTSF is too weak to induce a nonzero magnetization. However, as the temperature is progressively lowered, singlets with a distribution of effective exchange couplings are formed and a staggered field $H_{i, \perp}$ acting on them creates a distributed local magnetization of the form $\left\langle S_{i}^{\perp}\right\rangle \propto \mu_{\mathrm{B}} H_{i, \perp} / J_{i}$. Therefore, the histograms in Fig. 6(c) split and broaden with decreasing temperature. The symmetrical splitting reflects the alternating sign of the LTSF from site to site, with a mean absolute value of the local moment which progressively shifts away from $\left|\left\langle S_{i}^{\perp}\right\rangle\right|=0$.

In order to compare the simulated local-magnetization profiles with the NMR line shapes, structural details have to be considered. Eight configurations of disorder in systems with $L=6000$ sites were simulated. In order to reproduce 6000 unit cells, the local moments were then arranged into four different chains, with two inequivalent copper atoms each. At this first stage, the dipolar coupling was neglected, but we considered the hyperfine coupling to both the longitudinal and the transverse magnetization. Following the notation of Ref. 13, for $\mathbf{H} \| b$, the local hyperfine field $h_{\text {loc }}$ at a general ${ }^{29} \mathrm{Si}$ nuclear site can be written as

$$
h_{\mathrm{loc}}=\sum_{i=1}^{4}\left(a_{i}\left\langle S_{i}^{\|}\right\rangle+b_{i}\left\langle S_{i}^{\perp}\right\rangle\right),
$$

where the index $i$ runs over the four nearest-neighbor copper sites. With this notation, the relative NMR resonance frequency ${ }^{29} \omega$ can be written as ${ }^{29} \omega \approx \gamma h_{\mathrm{loc}}+\sigma_{b}$, with $\gamma$ as the ${ }^{29} \mathrm{Si}$ nuclear gyromagnetic ratio. From our previous study ${ }^{13}$ of $\mathrm{BaCu}_{2} \mathrm{Si}_{2} \mathrm{O}_{7}$ we can fix the parameters $\sum_{i} a_{i} \simeq-0.16 \mathrm{~T} / \mu_{\mathrm{B}}$, $\sum_{i}(-1)^{i} b_{i} \simeq 0.128 \mathrm{~T} / \mu_{\mathrm{B}}$, whereas $\sigma_{b} \simeq 0.018 \mathrm{MHz}$ at $7 \mathrm{~T}$. Each of the above hyperfine parameters, which couple the silicon nuclear magnetism to the longitudinal and transverse electronic magnetization of copper, represents the sum of four individual copper-to-silicon couplings. Given the broken translational symmetry due to disorder, the individual $\mathrm{Cu}-\mathrm{Si}$ couplings are essential for a quantitative comparison between data and theory. Unfortunately, these cannot be evaluated by experiment, leaving six unknown free parameters in the model.

For a comparison of the simulated NMR lines with data recorded at $5 \mathrm{~K}$, we used the following parameters (in $\mathrm{T} / \mu_{\mathrm{B}}$ units): $b_{1}=b_{3}=-0.24, b_{2}=-0.07$ for the coupling to the transverse magnetization, and $a_{1}=-0.16, a_{2}=0.08, a_{3}=$ -0.015 for the coupling to the longitudinal one. The sign of the parameters $b_{i}$ changes according to the considered Si nucleus. By making use of them and with no other free parameters, we succeeded in simulating the complete temperature dependence of the NMR lines, which account for the simultaneous presence of both an LTSF and a uniform magnetic field. The result is shown in Fig. 6(d), where the agreement between data and the theoretical model is again remarkable.

To be more specific, for a comparison with the experiment, we calculated the line shapes by convoluting the distribution of local fields at the ${ }^{29} \mathrm{Si}$ sites with a $2-\mathrm{kHz}$-wide Gaussian, which is equivalent to the width of the line profile of pure $\mathrm{BaCu}_{2} \mathrm{Si}_{2} \mathrm{O}_{7}$, shown in the inset of Fig. 5. The choice of the optimal binning range, which strongly affects the shape of the tails of the $S_{i}^{z}$ histograms in Fig. 6, was done by employing the algorithms proposed in Refs. 34 and 35. Note that the lines displayed in Fig. 6(d) show that the averaging process over four $\mathrm{Cu}$ sites removes the splitting in the original histograms in Fig. 6(c). In addition, the simulated lines shift with temperature, as indeed observed in the experiment. The minimally shifted line shape at $T=300 \mathrm{~K}$ reflects magnetic disorder. By comparing Figs. 6(b) and 6(d), it may be seen that the inclusion of a LTSF in the simulations significantly improves the agreement between theory and experiment. Therefore, from both a macroscopic and a local point of view, NMR data confirm that the static magnetic properties of $\mathrm{BaCu}_{2} \mathrm{SiGeO}_{7}$ are those of a RHC, with the addition of a residual LTSF.

To cross-check the consistency of the proposed model, it seems natural to consider the LTSF-induced effects also in the magnetometry data. Since the influence of the LTSF grows with the applied field $H$, we consider the calculated $M(H)$ curves (shown by dotted lines in Fig. 7) at two representative temperatures: $T=2$ and $10 \mathrm{~K}$. The calculation of $M(H)$ at $T=2 \mathrm{~K}$, using the average exchange coupling $J=$ $37 \mathrm{meV}$, reflects the linear behavior expected for an isotropic Heisenberg chain in its LL regime ${ }^{26}$ and differs substantially from the experimental data. In fact, this oversimplified model completely neglects the field-induced alignment of the still uncompensated spins that, in a random Heisenberg chain,

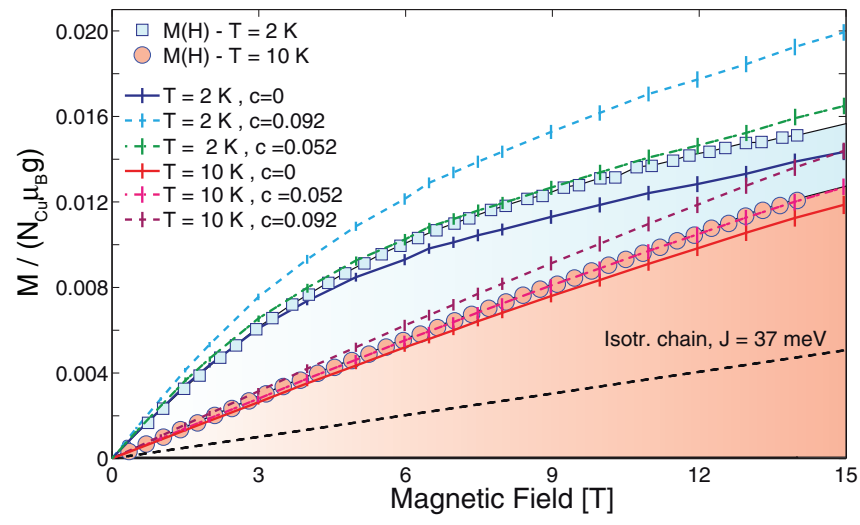

FIG. 7. (Color online) Field dependence of the magnetization of $\mathrm{BaCu}_{2} \mathrm{SiGeO}_{7}$ at 2 and $10 \mathrm{~K}$ (dots) and comparison with QMC simulations of the $J_{1}-J_{2}$ RHC model without $(c=0)$ and with $(c \neq 0)$ LTSF. The $c$ coefficient refers to the staggered field value $H_{i, \perp}=(-1)^{i} c H$, with $H$ the uniform field. Also shown is the field dependence of the ground-state magnetization $(T=0 \mathrm{~K})$ of an isotropic Heisenberg spin- $\frac{1}{2}$ chain, as from Ref. 26. The magnetization is expressed in normalized units ( $N$ is the total number of copper sites and $\mu_{\mathrm{B}}$ is the Bohr magneton), assuming $g=2$. 
survive even at the lowest temperatures. On the other hand, QMC simulations using the $J_{1}-J_{2}$ model discussed in Sec. III and in Ref. 15 show significant improvements over the simplified "average- $J$ " model (solid blue and red lines in Fig. 7 for $T=2$ and $10 \mathrm{~K}$, respectively).

The next refinement was to include both a uniform and a staggered magnetization in the $J_{1}-J_{2}$ model and combine them ${ }^{27}$ to obtain

$$
M(H, T)=\sum_{i}\left[\left\langle S_{i}^{\|}\right\rangle(H, T)+(-1)^{i} c\left\langle S_{i}^{\perp}\right\rangle\left(H_{i, \perp}, T\right)\right],
$$

with $\sum_{i}\left\langle S_{i}^{\|}\right\rangle(H, T)$ the RHC magnetization in a uniform field. As can be seen in Fig. 7, the QMC calculation for $M(H, T)$ with $c=0.092$ unexpectedly fails to reproduce the data. A good agreement with the magnetization scans at both temperatures was, however, obtained using $c \simeq 0.052$. The discrepancy in the prefactor is most probably due to the fact that the current model neglects the effects of structural disorder in $\mathrm{BaCu}_{2} \mathrm{SiGeO}_{7}$. A more refined model taking into account such subtleties, by including a site-dependent $c$ value, was not implemented in the present analysis. Nonetheless, the essence of RHC physics, which dominates the magnetic properties of the probed system, is borne out by the combined investigation of bulk magnetometry, NMR measurements, and QMC simulations. Taken together, they strongly suggest that $\mathrm{BaCu}_{2} \mathrm{SiGeO}_{7}$ is an excellent realization of a random Heisenberg chain model, as originally claimed in Ref. 15, thus providing the ideal testing ground for analytic theories modeling a random-singlet ground state.

\section{SUMMARY AND CONCLUSIONS}

In conclusion, we considered the $\mathrm{BaCu}_{2} \mathrm{SiGeO}_{7}$ compound, where spin- $\frac{1}{2} \mathrm{Cu}^{2+}$ ions interact via random exchange couplings, as a representative of RHC systems with bond disorder. We demonstrate that this spin-chain material has a ground state which is very well described on the basis of the RHC Hamiltonian in Eq. (1).

By using temperature- and magnetic field-dependent microscopic ${ }^{29}$ Si NMR measurements, combined with macroscopic magnetic-susceptibility data and results of detailed numerical quantum Monte Carlo simulations, we find a coherent description of the physical properties of the system, compatible with that of a RS phase. In particular, the considerable broadening of the NMR lines and the divergent magnetic response at low temperatures, combined with a nonlinear increase of the magnetization as a function of field at low $T$, all reflect the presence of unpaired spins with arbitrary small couplings even at temperatures close to zero.

Specific refinements in the simulations of $J_{1}-J_{2}$ model have shown that the addition of a local transverse staggered field LTSF (similar to that present in the parent compound
$\mathrm{BaCu}_{2} \mathrm{Si}_{2} \mathrm{O}_{7}$ ) (Ref. 13) is essential also for reproducing the observed NMR line shifts.

Since the magnetization of a singlet in a LTSF is proportional to the local field, the detection of the field distribution by future ${ }^{63} \mathrm{Cu}$ NMR measurements may provide a direct way to explore the $J$ distribution in a RHC. In addition, aside from the existing stretched-exponential NMR relaxation data, ${ }^{15}$ the spectral properties of random Heisenberg chains would be more directly accessible if neutron-scattering experiments in a lower-energy range would be possible.

\section{ACKNOWLEDGMENTS}

The authors thank A. Feiguin (Northwestern University, Boston) and O. Zaharko (PSI, Villigen) for useful discussions. The $\mathrm{BaCu}_{2} \mathrm{SiGeO}_{7}$ samples used in this work were prepared in the early 2000s in the group of Professor K. Uchinokura at the University of Tokyo. This work was financially supported in part by the Schweizerische Nationalfonds zur Förderung der Wissenschaftlichen Forschung (SNF) and the NCCR research pool MaNEP of SNF.

\section{APPENDIX: ERROR EVALUATION IN THE QMC SIMULATIONS}

In order to correctly interpret the numerical results, it is necessary to mention the theoretical expectations for the error bars in QMC simulations (i.e., specific to this method), in relation to the self-averaging induced errors (i.e., specific to disorder). ${ }^{36}$ For a spin chain of length $L$, one can define the ratio $R_{X}(L)=\left(\overline{X^{2}}-\bar{X}^{2}\right) / \bar{X}^{2}$, where $X$ is any macroscopic variable subject to self-averaging and $\bar{X}$ denotes the same quantity averaged over the realizations of disorder. ${ }^{36}$ By using 104 "Monte Carlo updates" (the number of random updates of the system performed by the algorithm before a new configuration is generated) and $10^{5}$ independent Monte Carlo measurements in a system of 6000 spins, the relative error for $M(T, H)$ in low-temperature QMC simulations was found to be 10 times lower than $\sqrt{R_{X}(L)}$, the error due to the average on all the realizations of disorder. Therefore, in our case $\sqrt{R_{X}(L)}$ is the dominant simulation error. Whenever the correlation length is $\xi \sim \ln ^{2}\left(J_{0} / T\right) \ll L$, self-averaging can be justified based on the central-limit theorem. ${ }^{36}$ In case of critical systems, where the correlation length tends to diverge $(\xi \gg L)$, the only way to obtain meaningful results in the presence of disorder consists in increasing the system size $L$. By simulating systems with different spin-chain lengths $L$, we find that $\sqrt{R_{X}(L \rightarrow \infty)}=\sigma_{\chi} /(\bar{X} \sqrt{L})$, with $\sigma_{\chi}$ the standard deviation of the local spin susceptibility. This implies the correct evaluation of the thermodynamic limit even in the problematic $\xi \gg 1$ case. Incidentally, since $\sigma_{\chi}$ is smaller at higher temperatures and magnetic fields, this justifies the smaller error bars in these regions.

\footnotetext{
*fr.casola@gmail.com

${ }^{1}$ H. A. Bethe, Z. Phys. 71, 205 (1931); P. W. Anderson, Mater. Res. Bull. 8, 153 (1973).

${ }^{2}$ C. Dasgupta and S. K. Ma, Phys. Rev. B 22, 1305 (1980).

${ }^{3}$ D. S. Fisher, Phys. Rev. B 50, 3799 (1994).
}

${ }^{4}$ F. Iglói and C. Monthus, Phys. Rep. 412, 277 (2005).

${ }^{5}$ E. C. Andrade and M. Vojta, Eur. Phys. Lett. 97, 37007 (2012).

${ }^{6}$ Y.-C. Lin, R. Mélin, H. Rieger, and F. Iglói, Phys. Rev. B 68, 024424 (2003). 
${ }^{7}$ N. Laflorencie, D. Poilblanc, and M. Sigrist, Phys. Rev. B 71, 212403 (2005).

${ }^{8}$ A. Zheludev and T. Roscilde, arXiv:1305.1194.

${ }^{9}$ S. Ward, P. Bouillot, H. Ryll, K. Kiefer, K. W. Krämer, Ch. Rüegg, C. Kollath, and T. Giamarchi, J. Phys.: Condens. Matter 25, 014004 (2013).

${ }^{10}$ G. Simutis, S. Gvasaliya, M. Månsson, A. L. Chernyshev, A. Mohan, S. Singh, C. Hess, A. T. Savici, A. I. Kolesnikov, A. Piovano, T. Perring, I. Zaliznyak, B. Büchner, and A. Zheludev, Phys. Rev. Lett. 111, 067204 (2013).

${ }^{11}$ B. Bauer et al. (ALPS Collaboration), J. Stat. Mech. (2011) P05001.

${ }^{12}$ Y. Yamada, Z. Hiroi, and M. Takano, J. Solid State Chem. 156, 101 (2001).

${ }^{13}$ F. Casola, T. Shiroka, V. Glazkov, A. Feiguin, G. Dhalenne, A. Revcolevschi, A. Zheludev, H.-R. Ott, and J. Mesot, Phys. Rev. B 86, 165111 (2012).

${ }^{14}$ L. P. Kadanoff, Ann. Phys. (NY) 100, 359 (1976).

${ }^{15}$ T. Shiroka, F. Casola, V. Glazkov, A. Zheludev, K. Prša, H.-R. Ott, and J. Mesot, Phys. Rev. Lett. 106, 137202 (2011).

${ }^{16}$ M. Thede, F. Xiao, Ch. Baines, C. Landee, E. Morenzoni, and A. Zheludev, Phys. Rev. B 86, 180407(R) (2012).

${ }^{17}$ E. Yusuf and K. Yang, Phys. Rev. B 72, 020403(R) (2005).

${ }^{18}$ A. Zheludev, T. Masuda, G. Dhalenne, A. Revcolevschi, C. Frost, and T. Perring, Phys. Rev. B 75, 054409 (2007).

${ }^{19}$ J. C. Bonner and M. E. Fisher, Phys. Rev. 135, A640 (1964).

${ }^{20}$ S. Bertaina and R. Hayn, Phys. Rev. B 73, 212409 (2006).

${ }^{21}$ T. Masuda, A. Zheludev, K. Uchinokura, J. H. Chung, and S. Park, Phys. Rev. Lett. 93, 077206 (2004).
${ }^{22}$ M. Kenzelmann, A. Zheludev, S. Raymond, E. Ressouche, T. Masuda, P. Böni, K. Kakurai, I. Tsukada, K. Uchinokura, and R. Coldea, Phys. Rev. B 64, 054422 (2001).

${ }^{23}$ A. Joshi and Kun Yang, Phys. Rev. B 67, 174403 (2003).

${ }^{24}$ R. J. Birgeneau, M. Greven, M. A. Kastner, Y. S. Lee, B. O. Wells, Y. Endoh, K. Yamada, and G. Shirane, Phys. Rev. B 59, 13788 (1999).

${ }^{25}$ T. Masuda, A. Zheludev, K. Uchinokura, J. H. Chung, and S. Park, Phys. Rev. Lett. 96, 169908(E) (2006).

${ }^{26}$ D. C. Johnston, R. K. Kremer, M. Troyer, X. Wang, A. Klümper, S. L. Bud'ko, A. F. Panchula, and P. C. Canfield, Phys. Rev. B 61, 9558 (2000).

${ }^{27}$ I. Affleck and M. Oshikawa, Phys. Rev. B 60, 1038 (1999).

${ }^{28}$ N. Shibata and K. Ueda, J. Phys. Soc. Jpn. 70, 3690 (2001).

${ }^{29}$ E. Westerberg, A. Furusaki, M. Sigrist, and P. A. Lee, Phys. Rev. Lett. 75, 4302 (1995).

${ }^{30}$ F. Alet, S. Wessel, and M. Troyer, Phys. Rev. E 71, 036706 (2005).

${ }^{31}$ W. G. Clark, M. E. Hanson, F. Lefloch, and P. Ségransan, Rev. Sci. Instrum. 66, 2453 (1995).

${ }^{32}$ R. K. Harris, E. D. Becker, S. M. Cabral de Menezes, R. Goodfellow, and P. Grangers, Pure Appl. Chem. 73, 1795 (2001).

${ }^{33}$ S. Miyahara, J.-B. Fouet, S. R. Manmana, R. M. Noack, H. Mayaffre, I. Sheikin, C. Berthier, and F. Mila, Phys. Rev. B 75, 184402 (2007).

${ }^{34}$ H. Shimazaki and S. Shinomoto, Neural Comput. 19, 1503 (2007).

${ }^{35} \mathrm{H}$. Shimazaki and S. Shinomoto, in Advances in Neural Information Processing Systems, edited by B. Schölkopf, J. Platt, and T. Hoffman, Vol. 19 (MIT Press, Cambridge, MA, 2007), pp. 1289-1296.

${ }^{36}$ A. Aharony and A. B. Harris, Phys. Rev. Lett. 77, 3700 (1996). 